\title{
Neural Readaptation to Earth's Gravity Following Return From Space
}

\author{
RICHARD BOYLE, ${ }^{1,5}$ ALLEN F. MENSINGER, ${ }^{2}$ KAORU YOSHIDA, ${ }^{3}$ SHIRO USUI, ${ }^{4}$ ANTHONY INTRAVAIA, ${ }^{1}$ \\ TIMOTHY TRICAS, ${ }^{6}$ AND STEPHEN M. HIGHSTEIN ${ }^{2}$ \\ ${ }^{1}$ National Aeronautics and Space Administration, Ames Research Center, Moffett Field, California 94035; ${ }^{2}$ Department of \\ Otolaryngology, Washington University School of Medicine, St. Louis, Missouri 63110; ${ }^{3}$ Department of Physiology, \\ University of Tsukuba, Tsukuba 305-8575; ${ }^{4}$ Toyohashi University of Technology, Toyohashi 441-8580, Japan; ${ }^{5}$ Department \\ of Otolaryngology/Head-Neck Surgery, Oregon Health Sciences University, Portland, Oregon 97201; and ${ }^{6}$ Department of \\ Biological Sciences, Florida Institute of Technology, Melbourne, Florida 32901
}

Received 4 December 2000; accepted in final form 27 April 2001

\begin{abstract}
Boyle, Richard, Allen F. Mensinger, Kaoru Yoshida, Shiro Usui, Anthony Intravaia, Timothy Tricas, and Stephen M. Highstein. Neural readaptation to earth's gravity following return from space. $J$ Neurophysiol 86: 2118-2122, 2001. The consequence of exposure to microgravity on the otolith organs was studied by recording the responses of vestibular nerve afferents supplying the utricular otolith organ to inertial accelerations in four toadfish, Opsanus tau, sequentially for 5 days following two National Aeronautics and Space Administration shuttle orbital flights. Within the first day postflight, the magnitude of response to an applied translation was on average three times greater than for controls. The reduced gravitational acceleration in orbit apparently resulted in an upregulation of the sensitivity of utricular afferents. By $30 \mathrm{~h}$ postflight, responses were statistically similar to control. The time course of return to normal afferent sensitivity parallels the reported decrease in vestibular disorientation in astronauts following return from space.
\end{abstract}

\section{IN T R O D U C T I O N}

It is of fundamental importance that organisms remain orientated within their terrestrial environment. Vertebrates possess a gravitoinertial sensing system, the utricular and saccular otolith organs that sense the sum of inertial and gravitoinertial forces due to head translation and head tilt relative to gravitational vertical and transform the vector sum of the imposing accelerations into a neural code. Since inertial and gravitational accelerations are indistinguishable (Einstein's equivalency principle) (Einstein 1945), both forces act equally on the otolith maculae. This code is combined with angular acceleration signals obtained from the semicircular canals and with information derived from other sensory modalities to compute a central representation of the body in space called the gravitoinertial vector. Thus the CNS resolves the ambiguity of gravity and self-motion and thereby maintains balance and equilibrium under varying conditions.

Exposure to microgravity imposes an extreme condition to which the traveler must adapt. Many, if not most, human travelers experience some disorientation during the first few days in microgravity called space adaptation syndrome akin to terrestrial motion sickness (Reason and Brandt 1975). From the

Address for reprint requests: R. Boyle, Ames Research Center, M/S 239-11, NASA, Moffett Field, CA 94035-1000 (E-mail: rboyle@mail.arc.nasa.gov). earliest manned missions, it was evident that adjustments to the microgravity environment in-flight and on return to Earth's $1 \mathrm{~g}$ occur (Black et al. 1999; Reschke et al. 1994). These adaptation mechanisms are conjectural and range from neural to structural changes or both. We studied the neural readaptation to Earth's $1 \mathrm{~g}$ using electrophysiological techniques to measure the response characteristics of utricular nerve afferents in fish on return from an exposure to microgravity.

\section{METHODS}

Six oyster toadfish, Opsanus tau, weighing 150-700 g, were individually housed in seawater tanks aboard two NASA shuttles. Fish were returned to the laboratory where all experiments were performed within $\sim 10 \mathrm{~h}$ of the shuttle landing. Surgical procedures are similar to Boyle and Highstein (1990) and were performed in accordance with the American Physiological Society Animal Care Guidelines and approved by the Institutional Animal Care and Use Committee. Fish were anesthetized with MS222 (Sigma) and secured in a Plexiglas tank placed atop an experimental table. A craniotomy exposed the utricle and its afferent nerve. Intra- and extracellular potentials were recorded from individual afferents using glass microelectrodes $(2 \mathrm{M}$ $\mathrm{LiCl}_{2}$ ). Potentials were conventionally amplified filtered, displayed, and converted to standard pulses. The experimental apparatus allowed manual yaw rotation about Earth vertical and repositioning of animal for translational acceleration parallel to Earth horizon and/or static tilt with respect to gravity. Fish could be repositioned in a $360^{\circ}$ circle such that the translational acceleration was delivered along any direction in the horizontal head plane and the acceleration was specifically directed, e.g., nose-down (pitch) or side-down (roll). Static sensitivity of otolith afferents to gravity was observed both in control and postflight fish. Afferent recordings were typically compromised by the manual repositioning of the table, and thus no reliable values of magnitude and change of magnitude of static sensitivity were possible. Position and motion of the fish were sensed by linear and rotary potentiometers and led, along with the afferent nerve pulses, to a computer interface (CED 1401Plus) connected to a Macintosh computer. Spike2 acquisition software, and Igor (WaveMetrics) analysis package were used. Statistical comparisons used the nonparametric unpaired Mann-Whitney or paired Spearman rank correlation tests,

\footnotetext{
The costs of publication of this article were defrayed in part by the payment of page charges. The article must therefore be hereby marked "advertisement" in accordance with 18 U.S.C. Section 1734 solely to indicate this fact.
} 
and indicated levels of statistical probability are two-tailed values (Instat software package).

\section{R E S UL T S}

Four toadfish were flown on the STS-90 "Neurolab" mission (17 days) and two aboard the STS-95 mission (9 days); two fish survived Neurolab and both survived STS-95. Responses of utricular afferents to gravitational (tilt) and inertial (translation) accelerations were recorded from four flight fish.

Control responses were obtained from 32 utricular afferents in three fish. Figure 1 shows the firing rate response (top, in
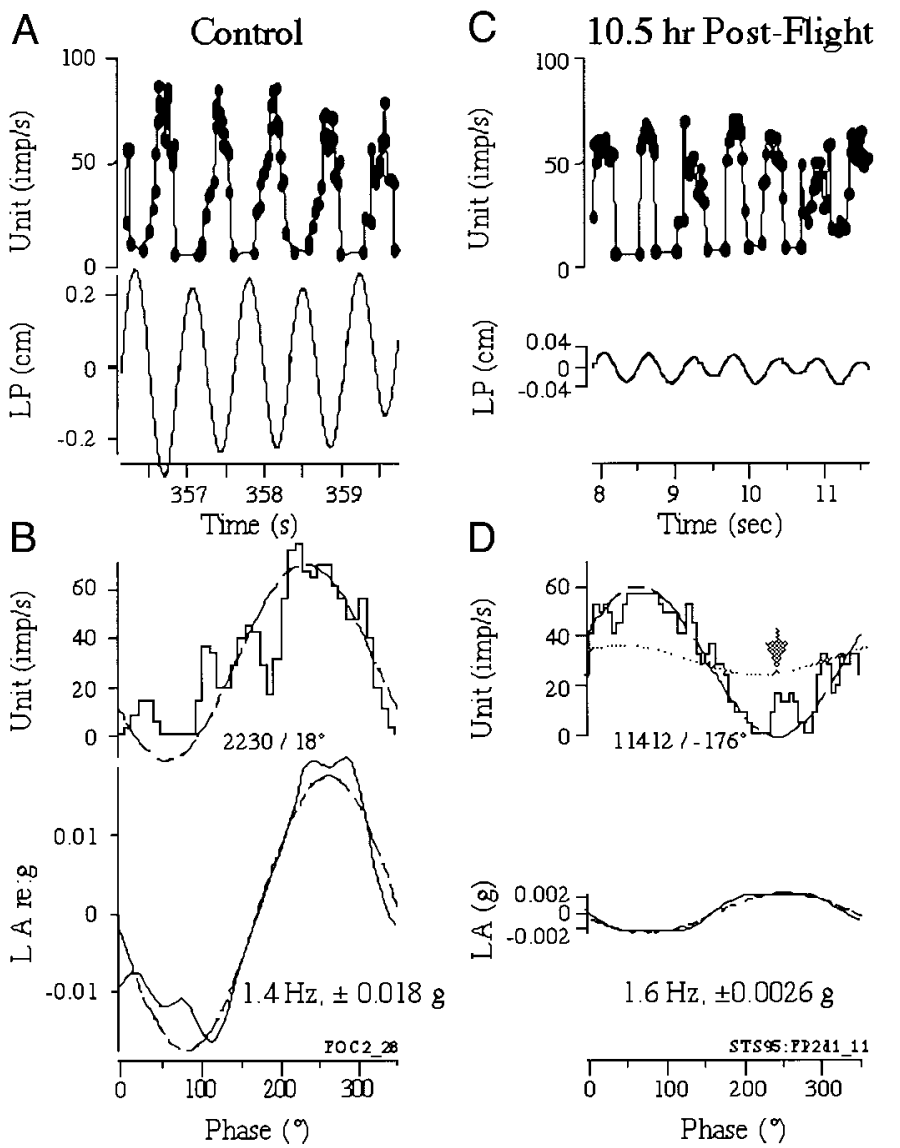

FIG. 1. Control ( $A$ and $B$ ) and $10.5 \mathrm{~h}$ postflight of STS-95 Shuttle $(C$ and $D)$ responses of toadfish utricular afferents to translational accelerations. $A$ and $C$ : afferent firing rate (in imp/s; top) is sinusoidally modulated to an applied sinusoidal change in linear position (LP, in $\mathrm{cm}$; bottom). In both records, the animals were rotated counter-clockwise by $90^{\circ}$ about the vertical axis resulting in a maximum rate increase for an acceleration directed rightward along the inter-labyrinth axis, ipsilateral to the recorded control afferent in $A$ and in the opposite sense for the postflight afferent in $C$. $B$ and $D$ : averaged response (top) of records shown in $A$ and $C$. Bottom: the averaged linear acceleration (LA re:g) of each stimulus. The complex-valued 1st harmonic of afferent response, represented as the dashed sinusoidal curve on the histogram, is divided by the complex-valued 1st harmonic of the stimulus, represented as the dashed sinusoidal curve on the linear acceleration trace, to determine the 1st harmonic sensitivity and phase of response. Ordinates in each panel are scaled equally to illustrate the primary finding: postflight afferents recorded shortly after return to Earth exhibit a profound hypersensitivity to translational accelerations. The amplitude of the applied $1.6-\mathrm{Hz}$ stimulus in $C$ and $D$ was almost negligible $( \pm 0.0026 \mathrm{~g}$ ) but induced a firing rate modulation of around $\pm 30 \mathrm{imp} / \mathrm{s}$ (or $11412 \mathrm{imp} \cdot \mathrm{s}^{-1} \cdot \mathrm{g}^{-1}$ ). An average control afferent would exhibit only about $\pm 4 \mathrm{imp} / \mathrm{s}$ modulation for this weak stimulus. The control response of the afferent shown in $A$ and $B$ is modeled as the dashed curve marked by the arrowhead in the histogram of $D$.
TABLE 1. Summary of control and postflight utricular afferent data

\begin{tabular}{lcc}
\hline \hline \multicolumn{1}{c}{ Fish } & Hours Post-Landing & $\begin{array}{c}\text { Mean Max. Sensitivity } \\
\text { imp } \cdot \mathrm{s}^{-1} \cdot g^{-1}\end{array}$ \\
\hline 3 controls & & $1,686 \pm 1,195$ \\
& & $(61-4,136 ; 32)$ \\
STS-95: $1+2$ & $10-16$ & $3,772 \pm 3,607 *$ \\
& & $(142-13,290 ; 24)$ \\
STS-95: 2 & $29.5-32$ & $1,582 \pm 1,750$ \\
& & $(101-4,992 ; 16)$ \\
STS-95: 2 & $52-55$ & $1,195 \pm 1,478$ \\
& & $(126-5,871 ; 18)$ \\
STS-90: 2 & $53-59$ & $1,399 \pm 1,599$ \\
& & $(1.16-7,819 ; 29)$ \\
STS-95: $1+2$ & $70-76$ & $1,337 \pm 1,076$ \\
& & $(100-4,738 ; 30)$ \\
STS-90: 3 & $112-117$ & $1,476 \pm 951$ \\
& & $(154-3,685 ; 28)$
\end{tabular}

Comparison of maximum response sensitivity (imp $\cdot \mathrm{s}^{-1} \cdot g^{-1}$ ) of utricular afferents recorded under control conditions to those at different time periods postflight of STS-90 ( 2 fish labeled 2 and 3 ) and STS-95 ( 2 fish labeled 1 and 2) shuttles. At 10-16 and $70-76 \mathrm{hr}$ postflight, the results obtained from the two STS-95 fish were comparable and are combined; the number of afferents are 8 (STS-95: 1) and 16 (STS-95: 2) at 10-16 hr and 10 (STS-95: 1) and 20 (STS-95: 2) at 70-76 hr. Mean \pm SD, with range of smallest to largest and number $(n)$ given in parentheses. Results show that the sensitivity was significantly greater than control $(P<0.01)$ for the earliest recording session $(10-16$ hr) from the two fish flown on STS-95; the results obtained from flight fish at later periods were not significantly different from control. The first records taken from fish flown on STS-90 began $53 \mathrm{hr}$ postflight after readaptation to Earth's gravity. $* P<0.01$.

$\mathrm{imp} / \mathrm{s})$ to a sinusoidal change in linear position (LP, bottom). In Fig. 1A, the fish was first rotated about the vertical axis in a counter-clockwise step to a $90^{\circ}$ head (and body) angle. The resulting translational acceleration (LA, Fig. $1 B$ ) was directed to the right, and ipsilateral to the recorded afferent, along the inter-labyrinth axis, and maximally excited the afferent. The averaged response to five stimulus cycles had a maximal sensitivity of $2,230 \mathrm{imp} \cdot \mathrm{s}^{-1} \cdot g^{-1}$ (Fig. $1 B$; see Table 1 ).

Hair cell bundles are morphologically polarized (Wersäll 1956); and hair cell's receptor potentials are directionally sensitive to bundle displacement (Hudspeth and Corey 1977; Shotwell et al. 1981). Directional selectivity of utricular afferents are distributed in a fanlike shape (Fernández and Goldberg 1976b) as expected from hair cell orientations in the utricular macula (Spoendlin 1966). Figure 2 shows the test used to determine the directional selectivity of individual afferents in control $(A)$ and postflight $(B)$ fish. A sinusoidal translational acceleration along an Earth-parallel plane was delivered at successive $15^{\circ}$ positions after the animal was stepped around a $360^{\circ}$ circle. Head angle $\left(^{\circ}\right)$ was defined using a right-hand rule relative to the laboratory: a positive acceleration at $0^{\circ}$ represents a forward movement directed out the animal's snout and one at $90^{\circ}$ a movement directed out the animal's right ear. Directional selectivity was determined by plotting the response sensitivity and phase relative to head angle. The data were fit by a rectified, cosine function (dashed lines in Fig. 2, $A$ and $B$ ), and correspondence between the tested and predicted responses reflects the sharpness of directional tuning. Control afferent in Fig. $2 A$ was sharply tuned and directionally selective to acceleration directed along the inter-labyrinth axis. All control afferents were directionally selective, and the maximal response vectors spanned $360^{\circ}$ of head angle.

In early postflight fish utricular afferents were hypersensitive 
A Control

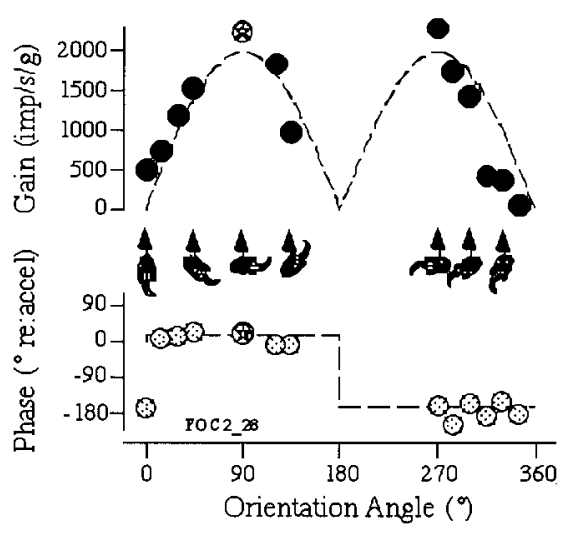

B $11 \mathrm{hr}$ Post-Flight

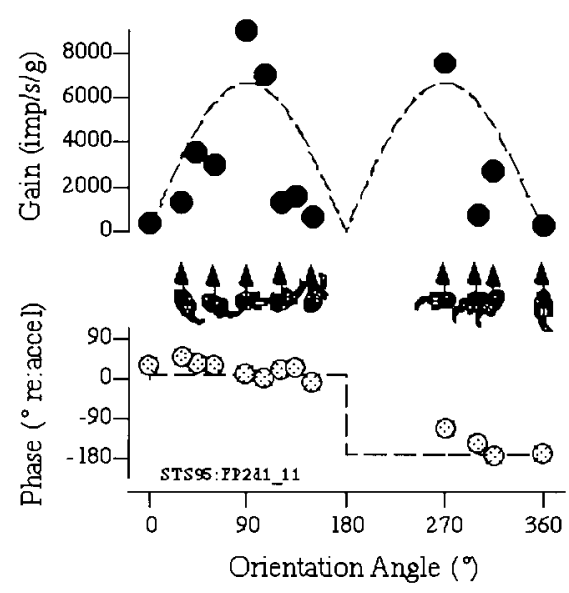

FIG. 2. Directional selectivity of control $(A)$ and $11 \mathrm{~h}$ postflight of STS-95 Shuttle $(B)$ toadfish utricular afferents to translational accelerations. $A$ and $B$ : sensitivity (solid symbols) and phase (open symbols) are plotted as a function of orientation angle (in ${ }^{\circ}$ ) of the animal. The small fish drawn between the sensitivity and phase plots graphically depict the animal's orientation as viewed from above for selected tests, and by convention forward motion of the test sled is denoted as an arrowhead. The individual response indicated by the star in panel $A$ represents the response shown in Fig. 1, $A$ and $B$. Forward acceleration out the animal's snout is given as $0^{\circ}$, and the animal is rotated counter-clockwise about the Earth's vertical axis and the designated angles follow a right-hand rule. For example, $90^{\circ}$ is an acceleration directed rightward along the inter-labyrinth axis, $180^{\circ}$ is a backward acceleration out the animal's tail, and $270^{\circ}$ is an acceleration directed leftward along the inter-labyrinth axis. The response of utricular afferents follows a rectified, cosine function (dashed curves fit to the empirical data) with respect to orientation angle, indicating directional selectivity. $A$ : control afferent. $B$ : afferent recorded $11 \mathrm{~h}$ postflight of STS-95 shuttle. Afferent exhibited an enhanced sensitivity to applied linear accelerations and directional selectivity.

to translational acceleration (Figs. 1-3) and directionally selective (Fig. 2B). One the first afferents recorded at $10.5 \mathrm{~h}$ postflight illustrates the striking increase in response sensitivity when stimulated at $\pm 0.0026 \mathrm{~g}$ acceleration or $\pm 0.025 \mathrm{~cm}$ displacement (Fig. 1, $C$ and $D$ ). The discharge modulation was about $\pm 30 \mathrm{imp} / \mathrm{s}$, yielding an afferent maximal sensitivity was $11,412 \mathrm{imp} \cdot \mathrm{s}^{-1} g^{-1}$, nearly sevenfold greater than the control mean and about threefold greater than the maximum response obtained in any individual control afferent $\left(4,136 \mathrm{imp} \cdot \mathrm{s}^{-1} \mathrm{~g}^{-1}\right.$; Table 1).

Data for both STS-90 and STS-95 shuttle missions are presented in the form of a probability plot (Fig. 3) to show the initial increase and recovery of response sensitivity. The data in this figure and in Table 1 are divided into groups based on time postflight. Maximum sensitivity of each afferent is plotted as a percentage of population sensitivity whose value is less than the individual sensitivity. For $\sim 60 \%$ of the afferents (14/24) in both fish on STS-95 (filled circles), labeled STS-95: $1+2$, the sensitivity recorded $10-16 \mathrm{~h}$ postflight was dramatically enhanced relative to control (crosses). Within this time group, the afferent sensitivity of the entire sample $(n=24)$ was roughly triple that in control $(P<0.01)$. The afferent sensitivity returns to near normal at the recorded time of $29.5-32 \mathrm{~h}$ postflight and remains within normal range after 5 days postflight.

To examine for possible recording bias, all measured parameters were compared between control and postflight afferents. No statistical difference was found in the range and mean of afferent discharge rate (imp/s) and regularity of discharge (standard deviation of the interval divided by the mean interspike interval) between postflight and control fish. An equal distribution of head angles evoking maximum and minimum response modulations of utricular afferents in postflight, similar to that observed in control fish, and response phase $\left(^{\circ}\right)$ were also found.

\section{I S C U S S I O N}

The increase in sensitivity of certain otolith afferents following space travel is most likely due to exposure of the animal to microgravity. The results, however, should be regarded as

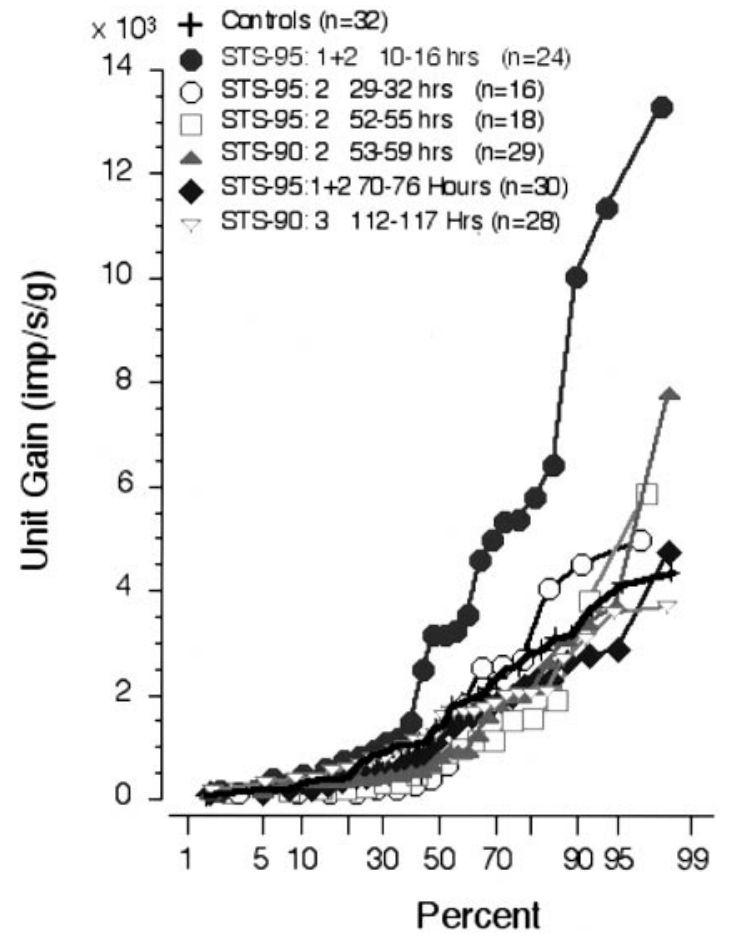

FIG. 3. Hypersensitivity of toadfish utricular afferents to translational accelerations within the first day postflight of STS-90 and STS-95 shuttles. Unit sensitivity (in imp $\cdot \mathrm{s}^{-1} g^{-1}$ ) of individual responses is plotted as a function of percent within each designated group. Groups are formed with respect to the time postflight, from the earliest time of $10-16 \mathrm{~h}$ for both fish $(1+2)$ of STS-95 (filled circles) to the latest time of $112-117 \mathrm{~h}$ for fish labeled 3 of STS-90 (inverted open triangle). Note that $\sim 60 \%$ of the afferents recorded in the 1st session (filled circles) reveal a marked sensitivity enhancement above control (black crosses). 
preliminary as single afferents were not studied sequentially. The following are speculations concerning potential mechanisms: an increase in transducer sensitivity, a temporary structural alteration affecting the mechanoreception of the otolith, otolith-stereociliary coupling causing enhanced bundle deflection for a given movement, or a pre- or postsynaptic alteration in the strength of synaptic transmission. The number of synaptic ribbons in certain type II hair cells in rodent is labile, increasing following exposure to microgravity (Ross 1993, 1994, 2000). Toadfish possess only type II hair cells and the afferent number of synaptic boutons and its response sensitivity to vestibular stimulation are correlated (Boyle et al. 1991). Thus an increase in number of synaptic ribbons in toadfish otolith hair cells following exposure to microgravity could potentially explain the present results.

If the otolith structure is arranged for optimal responses in 1 $g$, mechanical alterations may also occur in microgravity. For example, loss of gravitational force might displace the otolith relative to the macula, thus affecting neural responses. Altered gravity conditions might also trigger an adaptive response of the weight-lending structures (Wiederhold et al. 1997, 2000). Other reports suggest qualitative changes in otolith structure following space flight (Lychakov 1991; Susuki et al. 1993). However, Lim et al. (1974) and Sondag et al. (1995) could not demonstrate any change at the otoconial level after prolonged hypergravity by centrifugation. It is clear that more experiments under controlled states of altered gravity are required to determine the structural and developmental response of the otolith and the consequence of space flight on otolith function.

Adaptation of otolith hair cell receptor potentials occurs to prolonged deviation of their sensory hair bundle (Eatock et al. 1987; Fernández and Goldberg 1976a; Goldberg et al. 1990). Unweighting of the otolith mass in microgravity might potentially cause an adaptation of receptor potentials. That enhanced sensitivity remained for $\geq 24 \mathrm{~h}$ in toadfish afferents, substantially longer than the suggested time course of adaptation, is inconsistent with this view. Pioneering efforts at recording from otolith afferents in microgravity were performed by Bracchi et al. (1975) and Gualtierotti (1977); however, the results are difficult to interpret.

Otolith sensors provide a major input to the internal representation of the gravitoinertial vector. Thus an abnormal otolith component should have profound effects on the orientation of the organism and has been hypothesized to be causal in vestibular disorientation or space adaptation syndrome. The demonstrated time course in the altered otolith responses parallels the time course of disorientation experienced by space travelers and gives support to this hypothesis. In a complimentary study in another species of fish, the gain of an otolith-related vestiobuloocular reflex was significantly increased within the first postflight week, and returned to control levels in the second postflight week, of space missions (Sebastian et al. 2001).

The earliest recordings began $10 \mathrm{~h}$ after STS-95 landing, and to what extent this delay affects the interpretation of the data is indeterminate. Because of enhanced afferent sensitivity, the initial postflight results were limited to fewer stimulus frequencies $(1-2 \mathrm{~Hz})$ and lower amplitudes than delivered in control tests. These restrictions in stimulus parameters were required to minimize discharge nonlinearity (Boyle and Highstein 1990). Sensitivity on average declined from day 2 to 4 , and larger stimulus amplitudes could be progressively applied. The first single-unit recordings after the STS-90 flight began $53 \mathrm{~h}$ after landing, well after the postflight recovery time observed in the STS-95 fish. Significantly the directional tuning of afferents remained unchanged after exposure to microgravity. Therefore we would not expect significant remodeling of the spatial extent of dendritic arbors of afferents within the sensory epithelium to have occurred.

To date the toadfish utricular nerve has only been crudely evaluated at one frequency with a hand-powered linear sled. If the fish utricle bears any resemblance to similar epithelia studied in other species, more complete functional evaluations of afferents will no doubt demonstrate considerable diversity. It therefore remains to be tested whether a specific population of afferents demonstrated increased sensitivity or whether this finding is a general feature of all cells.

This work was supported by grants from National Aeronautics and Space Administration Ames (2-945), the National Institute on Deafness and Other Communication Disorders (PO-1 DC-01837), and the National Space Development Agency of Japan.

\section{REFERENCES}

Black FO, Paloski WH, Reschke MF, Igarashi M, Guedry F, And AnderSON DJ. Disruption of postural readaptation by inertial stimuli following space flight. J Vestibul Res 9: 369-378, 1999.

Boyle R, CAREY JP, AND HighSTEIN SM. Morphological correlates of response dynamics and efferent stimulation in horizontal semicircular canal afferents of the toadfish Opsanus tau. J Neurophysiol 66: 1504-1521, 1991.

Boyle R AND Highstein SM. Resting discharge and response dynamics of horizontal semicircular afferents of the toadfish Opsanus tau. J Neurosci 10: 1557-1569, 1990.

Bracchi F, Gualierotti T, Morabito A, and Rocca E. Multiday recordings from the primary neurons of the statoreceptors of the labyrinth of the bull frog. The effect of an extended period of "weightlessness" on the rate of firing at rest and in response to stimulation by brief periods of centrifugation (OFO-A orbiting experiment). Acta Otolaryngol Suppl 334: 1-27, 1975.

EATOCK RA, COREY DP, AND HudSPETH AJ. Adaptation of mechanoelectrical transduction in hair cells of the bullfrog's sacculus. J Neurosci 7: 282-236, 1987.

EINSTEIN A. Die Grundlage der allgemeinen Relativitätstheorie. Leipzig: Joh. Ambr. Barth, 1916. English version: The Meaning of Relativity. Princeton, NJ: Princeton Univ. Press, 1945.

FERnÁndez C AND GoldBerg JM. Physiology of peripheral neurons innervating otolith organs of the squirrel monkey. I. Response to static tilts and to long-duration centrifugal force. J Neurophysiol 39: 970-984, 1976a.

FERNÁNDEZ C AND GOLDBERG JM. Physiology of peripheral neurons innervating otolith organs of the squirrel monkey. II. Directional selectivity and force-response relations. J Neurophysiol 39: 985-995, 1976 b.

Goldberg JM, Desmadryl G, Baird RA, and Fernández C. The vestibular nerve of the chinchilla. IV. Discharge properties of utricular afferents. J Neurophysiol 63: 781-790, 1990.

GuALtierotTi T. The vestibular function research programme as a part of the Spacelab project: an investigation of integrated vestibular activity. Proc $R$ Soc Lond B Biol Sci 199: 493-503, 1977.

HUDSPETH AJ AND COREY DP. Sensitivity, polarity, and conductance change in the response of vertebrate hair cells to controlled mechanical stimuli. Proc Natl Acad Sci USA 74: 2407-2411, 1977.

Lim DJ, Stith JA, Stockwell CW, and Oyama J. Observations on saccules of rats exposed to long-term hypergravity. Aerospace Med 45: 705-710, 1974.

LYCHAKOV DV. Functional and adaptive changes in the vestibular apparatus in space flight. Physiologist 34, Suppl 1: S204-S205, 1991.

Reason JT And Brandt JJ. Motion Sickness. London: Academic, 1975.

Reschke MF, Bloomberg JJ, Paloski WH, Harm DL, and Parker DE. Neurophysiological aspects: sensory and sensory-motor function. In: Space Physiology and Medicine, edited by Nicogossian AE, Huntoon CL, and Pool SL. Philadelphia: Lea and Febiger, 1994, p. 261-285. 
Ross MD. Morphological changes in rat vestibular system following weightlessness. J Vestibul Res 3: 241-251, 1993.

Ross MD. A spaceflight study of synaptic plasticity in adult rat vestibular maculas. Acta Otolaryngol Suppl 516: 1-14, 1994.

Ross MD. Changes in ribbon synapses and rough endoplasmic reticulum of rat utricular macular hair cells in weightlessness. Acta Otolaryngol 120: 490499, 2000.

Sebastian C, Esseling K, And Horn E. Altered gravitational forces affect the development of the static vestibuloocular reflex in fish (Oreochromis mossambicus). J Neurobiol 46: 59-72, 2001.

Shotwell SL, Jacobs R, AND HudSPETH AJ. Directional sensitivity of individual vertebrate hair cells to controlled deflection of their hair bundles. Ann NY Acad Sci 374: 1-10, 1981.

Sondag HN, De Jong HA, Marle J, ANd Oosterveld WJ. Effects of sustained acceleration on the morphological properties of otoconia in hamsters. Acta Otolaryngol 115: 227-230, 1995.
SPOENDLIN HH. The ultrastructure of the vestibular sense organ. In: The Vestibular System and Its Diseases, edited by Wolfson RJ. Philadelphia, PA: University of Pennsylvania Press, 1966, p. 39-68.

Susuki M, HaRada Y, AND Sekitani T. Vestibular endorgan of the frog after the space flight and postural alteration of the neurectomized frogits morphological and functional resilience. J Vestibul Res 3: 253-258, 1993.

WERSÄLL J. Studies on structure and innervation of sensory epithelium of cristae ampullares in guinea pig; light and electron microscopic investigation. Acta Otolaryngol Suppl 126: 1-85, 1956.

Wiederhold ML, Harrison JL, Parker K, ANd Nomura H. Otoliths developed in microgravity. J Grav Physiol 7: P39-P42, 2000.

Wiederhold ML, Pedrozo HA, Harrison Jl, Hejl R, and Gao W. Development of gravity-sensing organs in altered gravity conditions: opposite conclusions from an amphibian and a molluscan preparation. $J$ Grav Physiol 4: P51-P54, 1997. 